\title{
Erratum to: Pediatric Postgraduate Training in India-Residents' Perspective
}

\author{
M. G. Geeta • P. Krishnakumar • A. Riyaz • \\ N. C. Cherian - A. V. Gopalan
}

Published online: 3 July 2014

(C) Dr. K C Chaudhuri Foundation 2014

Erratum to: Indian J Pediatr (May 2014) 81(5):521-522
DOI 10.1007/s12098-013-1308-0

In the article entitled "Pediatric Postgraduate Training in India

- Residents' Perspective" [Indian J Pediatrics (May 2014)
81(5):521-522] the following corrections are to be made in tables 1 and 2.

In table 1 Daily clinical class was available in 38(86 \%) Govt. medical colleges and not $36(86 \%)$ as mentioned in the paper.

Table 2 Comparison of perceptions and practice of pediatric residents from different institutions with regard to doctor-patient (parent) communication

\begin{tabular}{|c|c|c|c|c|c|}
\hline Questions & Over all No. (\%) & $\begin{array}{l}\text { Govt. medical } \\
\text { college }\end{array}$ & $\begin{array}{l}\text { Private } \\
\text { hospital }\end{array}$ & $\begin{array}{l}\text { Private medical } \\
\text { college }\end{array}$ & $P$ \\
\hline Satisfied as a pediatric resident & $45(50)$ & $22(50)$ & $14(52)$ & $9(53)$ & 0.975 \\
\hline Comfortable when breaking bad news & $31(34)$ & $14(32)$ & $9(33)$ & $8(44)$ & 0.626 \\
\hline Confident to deal with dissatisfied patients & $36(40)$ & $19(43)$ & $13(50)$ & $4(22)$ & 0.167 \\
\hline Feeling relaxed and stress free while seeing patients & $21(23)$ & $8(18)$ & $9(35)$ & $4(22)$ & 0.292 \\
\hline Importance of non verbal communication & $59(66)$ & $34(79)$ & $14(54)$ & $11(61)$ & 0.075 \\
\hline Introducing to the patients & $31(34)$ & $8(18)$ & $12(46)$ & $11(61)$ & 0.002 \\
\hline Dealing psychological problems of patients & $27(30)$ & $11(25)$ & $11(42)$ & $5(28)$ & 0.302 \\
\hline Using open questions & $45(50)$ & $22(51)$ & $17(65)$ & $6(33)$ & 0.112 \\
\hline Maintaining privacy & $79(88)$ & $36(82)$ & $25(96)$ & $18(100)$ & 0.044 \\
\hline Consider wishes of parents/patients & $69(77)$ & $32(73)$ & $23(89)$ & $14(78)$ & 0.302 \\
\hline
\end{tabular}

The online version of the original article can be found at http//dx.doi.org/ 10.1007/s12098-013-1308-0.

\footnotetext{
M. G. Geeta • P. Krishnakumar · A. Riyaz • N. C. Cherian •

A. V. Gopalan

Department of Pediatrics, Government Medical College, Kozhikode,

Kerala, India

P. Krishnakumar $(\square)$

Child Development Services, Institute of Mental Health and

Neurosciences, Kozhikode, Kerala 673008, India

e-mail: krikurp@gmail.com
} 